\title{
(4)
}

\section{GESTIÓN MUNICIPAL DEL AGUA EN GUATEMALA: CO-RESPONSABILIDAD LOCAL ANTE UN ESTADO AUSTENTE. BALANCE Y LECCIONES DE LA EXPERIENCIA DE PROMUDEL.}

\author{
Esteban Valenzuela Van Treek. ${ }^{24}$
}

\section{RESUMEN}

La principal competencia de los municipios guatemaltecos es gestionar el agua y clorarla, lo que ocurre de manera deficitaria, generando una alta contaminación, y enfermedades por diarreas, desnutrición y mortalidad infantil. Las razones para esta crítica gestión municipal del agua se relacionan con la ausencia del Estado en el sector: no hay superintendencia sanitaria, ni programas regionalizados de inversión en el ciclo del agua, ni sanciones a los municipios que no cloran sus sistemas. La alta corrupción política y baja profesionalización municipal, generan un contexto crítico. Por su parte, las comunidades mayas tienen sistemas ancestrales sin apoyo estatal. El artículo explica esta realidad, muestra los resultados del Programa de Municipios por el Desarrollo Local (Promudel) en cuarenta municipios y propone la realización de cambios fiscales, organizacionales y político-culturales para hacer real la gobernabilidad municipal del agua.

Palabras claves: agua potable, gobernabilidad, Guatemala, mayas, municipios.

\section{ABSTRACT}

The main responsibility of Guatemalan municipalities is to manage and chlorinate the water, what happens in a deficient way, generating high pollution, diarrheal diseases, malnutrition and infant mortality. The reasons for this poor municipal water management are the absence of the state rule, medical supervision, regional programs for investment in water and sanctions for municipalities that do not chlorinate their systems. The high political corruption and low professionalism municipal generate a critical context. Furthermore, Mayan communities have ancestral systems without state support. The article explains this reality,

24 Director del Dpto. de Ciencia Política y Relaciones Internacionales de la U. Alberto Hurtado. Doctor en Historia de la U. de Valencia, Máster en C. Política de la P. U. Católica de Chile y Máster en Desarrollo de la U. Wisconsin-Madison. Fue alcalde y diputado por Rancagua. Creador de la Asociación Chilena de Municipalidades con la Fundación Ebert. Ha sido asesor principal de la GIZ alemana en R. Dominicana, Colombia y Guatemala. 
shows the results of the Municipality Program for Local Development (Promudel) in forty municipalities, and proposes tax changes, organizational and political-cultural reforms for improving municipal water governance.

Key words: drinkable water, governance, Guatemala, Mayan, municipalities.

\section{EL REZAGO MUNICIPALISTA EN UN PAÍS EN RIESG025.}

Guatemala es un país en riesgo, con un desarrollo municipal medianobajo y con una crisis en su gestión del agua potable. La eficacia de la gestión local se da en el contexto de la cultura política global de un país. El de Guatemala es conocido por sus claroscuros. La fortaleza de los pueblos indígenas, donde hay mayor cohesión social y menos violencia que las zonas blancas, es una diferencia significativa: las regiones mayas tienen un promedio de 10 muertes violentas cada cien mil habitantes al año, en comparación a 60 de las zonas ladinas (blancas) del Istmo Centroamericano (México, Honduras, Guatemala, El Salvador), según las series de informes respaldados por la cooperación danesa (Programa Estado de la Nación, 2011).

Estos informes lo sitúan como un país en riesgo, por su Estado débil, alta pobreza, corrupción y bajos índices de desarrollo humano. De hecho, se ubica en el lugar 133 del Índice de Desarrollo Humano en la actualidad. (PNUD, 2012). Aunque el país ha mejorado los indicadores de salud, estos siguen siendo deficitarios, con una mortalidad infantil de 40 niños cada mil nacidos, solo mejor que Haití, Bolivia y Nicaragua, y muy por debajo del promedio regional de 15 (OPS, 2012). Una muestra de sus dificultades de gobernabilidad fue la instalación por parte de Naciones Unidas del Comité Internacional contra la Impunidad en Guatemala $(\mathrm{CIClG})$, con aceptación por los gobiernos de Bergé, Colom y Otto Pérez, el cual ha servido para combatir los mayores casos de impunidad y corrupción estatal.

La raíz de la desigualdad se encuentra en que es el país de más baja carga tributaria de toda América Latina, situándose en el $11 \%$ del PIB (el promedio de América es $20 \%$ y de los países desarrollados, 32\%); es incluso más bajo que Haití, que es 12\% (ILPES, 2011).

Los municipios son el principal espacio de la descentralización estatal, con un bajo poder, ya que dependen del situado constitucional que es una transferencia monetaria desde los ingresos totales del Gobierno Central, ubicando a Guatemala entre los países cuyos entes subnacionales participan de menos del $10 \%$ de los ingresos totales de la nación (Rosales, 2012). En el cuadro comparativo, elaborado por Rosales en base a datos del Fondo Monetario Internacional (FMI), Banco Mundial y Banco Interamericano del Desarrollo (BID), Guatemala baja del $4.5 \%$ de participación de los entes subnacionales en el gasto fiscal total en 1980 a un $4.4 \%$ el 2009 , desacoplado de la ma-

25 Este artículo es el resultado de cuatro investigaciones del autor para Agencia de Cooperación Alemana (GIZ) Guatemala: Políticas públicas y competencias municipales (2010), Gobernabilidad del agua en Guatemala (2011), Administración municipal en Guatemala (2012) y Síntesis de Impactos de las políticas públicas en agua en los 40 municipios asesorados por GIZ-PROMUDEL (2012). 
yor descentralización que se da en el Continente, que creció del $11.6 \%$ al $19 \%$. Es decir, hay un retroceso significativo, ya que en la década de 1980 el país se acercaba a la mitad del gasto promedio de los demás países en sus entes subnacionales, mientras que ahora Guatemala destina menos de un tercio del promedio regional. No obstante, se puede destacar la gestión en la ciudad de Guatemala y otros municipios de la zona metropolitana, así como la turística Antigua, que han roto el círculo de la pereza fiscal, cobrando el impuesto predial, lo que les ha permitido mejorar sus servicios a la población.

Sin embargo, el ambiente subjetivo en el que se desenvuelven los municipios es adverso, marcado por la alta criminalidad y corrupción. La existencia de poderes paralelos al Estado, mafias y redes económicas controladas en su mayoría por el narcotráfico, generan la penetración y cooptación de niveles estatales y un clima generalizado de extorsión, amenazas e impunidad. En este cuadro, no es posible asegurar el funcionamiento normal de los municipios, muchos de los cuales, por ejemplo, para lograr adjudicarse proyectos desde agencias nacionales y fondos departamentales (regiones), deben aceptar malas prácticas y hacer un extensivo uso de consultoras ligadas a los mismos parlamentarios y políticos. Entre estas malas prácticas se encuentra la llamada obra gris, para cuya obtención se privilegian los contratos de mejoras de caminos e infraestructura, dejando de lado la inversión en servicios públicos esenciales como el agua, que implicarían una mayor complejidad para robar. Un periódico reseña estas malas prácticas, cuestionadas por el director de Acción Ciudadana (Transparencia Internacional en Guatemala), Manfredo Marroquín:

"Pero pese a ser un secreto a voces, pocos ciudadanos conocen a profundidad cómo 'se manejan' las obras públicas y el dinero ilícito que reciben los diputados con el objetivo de beneficiar a grupos específicos, a través del Listado Geográfico de Obras. Las comunidades representadas en los 22 Concejos Departamentales de Desarrollo (Codede) y las instituciones de gobierno formulan propuestas para la construcción de proyectos -alcantarillados, parques y escuelas, por ejemplo- en el Sistema Nacional de Inversión. La Secretaría de Planificación y Programación de la Presidencia evalúa los proyectos, los integra al Programa de Inversión Pública y los remite al Ministerio de Finanzas Públicas, que a su vez prepara el Programa de Inversión Física, Financiera y Transferencias, el cual los adjunta cada año al proyecto de presupuesto.

Sin embargo, este ejercicio democrático-que involucra a las comunidades y al gobierno- se interrumpe cuando el proyecto anual del presupuesto llega a manos de los diputados, en el Congreso, y las obras públicas que se planificaron en un proceso largo y cuidadoso ya no se priorizan según las necesidades de la población, sino que dependen de los intereses de los legisladores quienes modifican el Listado Geográfico de Obras a su conveniencia."26 
La falta de control y cumplimiento de la ley hace vano el trabajo legislativo. La ausencia de un diseño institucional básico, como la supervigilancia de la calidad del agua potable con sanciones efectivas ante las infracciones que se puedan cometer, hace que los decretos alcaldicios se vuelvan retóricos. Además, la falta de independencia y probidad en la Contraloría General de Cuentas de Guatemala, agrava la falta de cumplimiento de la legalidad (law enforcement). El pago de impuestos claves, como el Impuesto Único sobre Inmuebles (IUSI), es optativo y vago. Falta profesionalización en las superintendencias de servicios básicos y control de legalidad.

En suma: pocos recursos, frágil división de poderes, institucionalidad débil y falta de preparación del recurso humano, así como muchos organismos con tareas genéricas que no se materializan en resultados tangibles. El Estado es débil y poco profesionalizado. La labor de los Departamentos es prácticamente nula, como lo muestra el Informe 2011 del PNUD de Guatemala, en relación a la presencia estatal en los territorios (PNUD, 2011). No se cumplió la meta de los Acuerdos de Paz de 1998, firmados tras el cruento conflicto interno que costó la vida a 200 mil guatemaltecos, especialmente en el lapso 1980-1984. Uno de los acuerdos fue aumentar la carga tributaria y tal como se explicó, éste se sitúa en solo un $10 \%$ en el último quinquenio.
Las propuestas son evidentes y profusamente promocionadas por los organismos internacionales (Carías, 2011), pero no fructifican en el Congreso. Estas van desde terminar las numerosas exenciones, hasta hacer obligatorio el impuesto predial; aumentar los impuestos a la renta y el royalty a las empresas mineras y petroleras; o reponer el tributo a las nuevas construcciones, que, de manera insólita, se eliminó en la última reforma al Código Municipal (Tavico, 2010).

\section{LA CRÍTICA SITUACIÓN DE LOS SERVI- CIOS DE AGUA MUNICIPALES: ENTRE EL ESTADO AUSENTE Y LA AUSENCIA DE CO- RESPONSABILIDAD LOCAL}

"Gastamos más en trofeos y pelotas que en clorar el agua y salvar vidas." (Alcalde de Raxruhá, marzo, 2011)27

La expresión del alcalde de Raxruhá es exagerada, pero muestra una verdad: el agua es un problema privatizado en Guatemala. Cada comunidad se las arregla como pueda, la empresa de botellas de agua Salvavidas inunda el mercado, las empresas locales de purificación del agua se multiplican. Todo ello ante sistemas municipales mal mantenidos, contaminados y discontinuados en las cabeceras urbanas.

Los municipios invierten buena parte de sus presupuestos en competencias que formalmente son del Estado central guatemalteco, especialmente en la contratación de profesores y el mantenimiento de caminos rurales. Lo último es esencial para no aislar las comunidades, en un país de alta ocu-

27 Palabras vertidas en el contexto del debate de la política en agua y los resultados del ranking de PROMUDE. Ver: http:// www.promudel.org.gt/ Muni - Noticias / Alta Verapaz / Políticas Públicas / Ecos Locales. 
rrencia de tormentas y deslizamientos de terrenos. Las comunidades, organizadas en Consejos Comunitarios de Desarrollo (Cocode) suelen demandar estas problemáticas, sumadas a las de apoyo en temas de producción y seguridad alimentaria. Esto sucede especialmente en el altiplano guatemalteco, donde viven cinco millones de mayas. En consecuencia, las municipalidades no logran concentrarse en las competencias que les son propias, entre ellas, la atención a los servicios públicos en su jurisdicción, dentro de los cuales se encuentran, principalmente, el abastecimiento de agua potable debidamente clorada, alcantarillado y la recolección, tratamiento y disposición final de residuos y desechos sólidos. ${ }^{28}$

En Guatemala, desde la colonia los municipios han tenido algún vínculo con el suministro de agua, instalación de pilas en sus mercados y limpieza de los mataderos. No obstante, más allá de las disposiciones constitucionales, no ha habido supervisión estatal. La prestación de los servicios municipios se ha dado de manera independiente, sin ninguna regulación ni control por parte del gobierno central en cuanto a aspectos técnicos, comerciales y relaciones con los usuarios (Rodríguez, 2011).

El año 1985, a raíz de una iniciativa del gobierno central, se crea el Consejo Permanente de Coordinación de Agua y Saneamiento (Copecas). Luego de un análisis sectorial apoyado por la Organización Panamericana de la Salud (OPS), en 1995 se crea un Comité de Alto Nivel, con el fin de definir las políticas y acciones para reorganizar el sector. Como resultado de estas acciones, en 1997 se le encarga al Instituto de Fomento Municipal (Infom), la gestión de políticas y estrategias del sector y se aprueba el traspaso de la Unidad Ejecutora de Acueductos Rurales (Unepar) al Infom.

El año 2000 se disuelven el Copecas y el Comité de Alto Nivel y se crea la Comisión Intersectorial, encargada del proceso de reforma y modernización del sector. A su vez, esta comisión es disuelta el 2005 y en su reemplazo se crea, de manera temporal, la Comisión Nacional de Coordinación del Recurso Agua (Conagua), organismo que dependerá directamente de la Presidencia, cuya responsabilidad será la de impulsar la Política Hídrica Nacional. Hasta este momento, los esfuerzos por estructurar el Sector de Agua y Saneamiento de acuerdo a las necesidades del país no daban frutos. Ninguna de las instituciones involucradas en el proceso anterior, así como los Ministerios de Salud y Medio Ambiente, había tomado el liderazgo (Schifini, 2005: 4).

En un intento por superar estas limitaciones, el año 2008 se crea el Gabinete Específico del Agua, el cual tuvo como propósito la coordinación de acciones relacionadas específicamente a los servicios de agua y saneamiento (Lentini, 2010: 17). Así, en marzo del 2010, por Acuerdo Ministerial de Salud, se crea la Unidad Especial de Ejecución Administrativa para el Control de Agua Potable y Saneamiento con el propósito que "... de forma responsable, (vele por) el

28 Así lo establece el Código Municipal, en su artículo 68, inciso a). Los servicios pueden ser prestados de forma directa, mancomunada entre varios municipios o concesionados. 
desarrollo de los aspectos generales de políticas sectoriales, planificación sectorial, atribuciones de administración, coordinación, regulación, evaluación y otras pertinentes, se desarrollen en un proceso integral."${ }^{29}$

Esta es la historia formal. Pero, desde la gestión del Programa de Municipios por el Desarrollo Local (Promudel), en apoyo directo a cuarenta municipios de cuatro departamentos con alta concentración de población maya, se observó una ausencia significativa del Estado central: más de la mitad de las muestras de agua contaminadas y ningún alcalde sancionado por no clorar el agua (Promudel - GIZ, 2010, 2011, 2012). En el año 2013, el Colegio de Ingenieros concluyó que el $80 \%$ de los sistema tiene contaminación: "Un estudio efectuado por profesionales sanitarios del Colegio de Ingenieros revela que el 80 por ciento de las fuentes de agua del país está contaminado por bacterias y químicos, lo que contribuye a que cada año se incrementen los casos de enfermedades diarreicas".30

La ausencia de equipos profesionales expertos por regiones para gestionar proyectos de agua se hace evidente en los retrasos en ejecutar proyectos de donantes. Una parálisis por falta de Estado, corrupción y mala política. Por ejemplo, la Agencia Española de Cooperación (AECI), ha tenido serios problemas para ejecutar su fondo de apoyo a infraestructuras en agua por la falta de consenso en el Congreso, debido a que partidos oficialistas $y$ opositores tienen baja colaboración y buscan repartir políticamente los municipios beneficiarios. Además, de los problemas de ingeniería y ejecución de lo que se aprueba. En reuniones bilaterales se reconoce el atraso y los problemas de ejecución:

\begin{abstract}
"La vicemandataria Roxana Baldetti se reunió este jueves con el director para América Latina de la Agencia Española de Cooperación Internacional para el Desarrollo (AECID), Rafael Garranzo; el embajador de España en Guatemala, Manuel Lejarreta; el presidente de la Junta Directiva del Instituto de Fomento Municipal, Antonio Coro, y el titular de la Secretaría de Planificación y Programación Económica de la Presidencia (Segeplan), Fernando Carrera, con el fin de impulsar el proyecto de agua potable y saneamiento en las municipalidades, financiado por una donación española de $\$ 50$ millones (Q390 millones).
\end{abstract}

La reunión fue convocada debido a que el proyecto no marcha con la velocidad esperada, luego de que España otorgó el financiamiento hace ocho años y el plazo para la ejecución de dicho fondo vence en enero de 2015 $5^{1 / 31}$.

Un informe reciente de la Cepal - GTZ (2011) concluye que sólo el 15\% de los sistemas de agua en Guatemala son de calidad, presión debida y cloración adecuada. Los investigadores 
de la Universidad de San Carlos, Juan Francisco Pérez y Bessie Oliva, ambos licenciados en Química, son categóricos en las conclusiones en una investigación suya sobre el agua en Guatemala:

"País rico en recursos hídricos, entre los que se encuentran ríos y lagos con volumen suficiente para satisfacer las necesidades de agua potable por parte de la población y la demanda de la industria. Sin embargo, el $90 \%$ de las fuentes superficiales se encuentran contaminadas, lo cual ha incidido en que más de un $40 \%$ de la población no tenga acceso a agua potable y se tengan tasas de mortalidad infantil superiores $a$ un 40 por mil nacidos vivos, siendo un $24 \%$ de las muertes debido a enfermedades diarreicas. Las áreas marginales son las más afectadas, donde los infantes sufren las consecuencias, padeciendo retrasos en su desarrollo y un bajo nivel de vida. Entre las enfermedades relacionadas con la mala calidad del agua que tienen incidencia en Guatemala, se encuentran la diarrea, tifoidea, hepatitis, malaria y dengue. En diferentes estudios se ha determinado que los principales ríos y lagos del país, entre ellos el río Las Vacas, río Motagua, río Polochic, Lago Amatitlán, Lago Atitlán, Lago Izabal y Lago Petén Itzá, presentan niveles de contaminación que superan los valores guía para agua potable y agua para recreación." (Pérez y Oliva, 2008: $12-13)$.

En la única encuesta nacional conocida sobre el tema, realizada por la Secretaría General de Planificación Nacional (Segeplan), el año 2006, se colige la enorme fragilidad de los sistemas de agua alrededor de la zona metropolitana de ciudad de Guatemala. En los departamentos con población mayoritariamente de origen maya, más de un tercio de sus habitantes no tiene acceso a agua y la cloración es casi nula.

\section{Cuadro $\mathrm{n}^{\circ}$ 1. Porcentaje de Hogares con acceso a Agua, Departamento de Ciudad de Guatemala versus cuatro de mayoría maya.}

\begin{tabular}{|l|c|c|c|}
\multicolumn{1}{c}{ DEPARTAMENTO } & \multicolumn{1}{c}{$\begin{array}{c}\text { PORCENTAJE CON } \\
\text { AGUA EN LA CASA }\end{array}$} & \multicolumn{1}{c|}{$\begin{array}{c}\text { PORCENTAJE CON AGUA EN } \\
\text { LA CERCANÍA DEL HOGAR }\end{array}$} & $\begin{array}{c}\text { SIN ACCESO } \\
\text { CERCANO }\end{array}$ \\
\hline Ciudad de Guatemala & 82 & 8 & 10 \\
\hline Huehuetenango & 63 & 6 & 31 \\
\hline Quiché & 61 & 4 & 35 \\
\hline Alta Verapaz & 21 & 21 & 58 \\
\hline Baja Verapaz & 28 & 42 & 30 \\
\hline
\end{tabular}

Fuente: Encuesta Nacional de Condiciones de Vida (ENCOVI) 2006, supervisada por la Secretaria General de Planificación Nacional (Segeplan). Nota: esta medición no contabiliza la potabilización del agua. 


\section{MODELO DE GESTIÓN DEL SERVICIO DE AGUA PROPUESTO POR PROMUDEL Y SUS IMPACTOS: CORRESPONSABILIDAD ANTE EL ESTADO AUSENTE}

\section{III.1. La estrategia}

Promudel comenzó el año 2007 y culmina este año 2013, como un programa conjunto de los gobiernos de Alemania y Suecia. Gestionado por la Agencia de Cooperación Alemana (GIZ, ex GTZ). Tras un inicio marcado por un menú amplio de proyectos, el año 2008, su coordinador Thorsten Sagawe encabeza un viraje de la agencia encaminado hacia lograr que los municipios se entusiasmen con cumplir su principal tarea: mejorar los sistemas de agua, clorarlos y así bajar la desnutrición y la mortalidad infantil en las zonas mayas de extrema pobreza.

Con escepticismo hacia los cambios provenientes desde arriba, tales como la creación de una superintendencia sanitaria y la inversión de recursos territoriales bien focalizados en agua, la GIZ privilegió la asistencia directa a 40 municipios en departamentos de mayoría maya, buscando recobrar buenas prácticas por la vía de la transformación del $60 \%$ de estos en materia de: cobro de impuestos, rendición de cuentas, participación ciudadana, políticas públicas pro agua potable y creación de unidades técnicas para el servicio de aguas. Es decir, ante el Estado ausente, generar la confianza mutua municipio/ciudadanía, en un modelo de corresponsabilidad, para aumentar la movilización de recursos locales a favor de sistemas de agua de mejor calidad.

La estrategia consistió en la implementación de cinco instrumentos, que convergen en torno al mismo objetivo principal:

\section{a) Mejorar las finanzas locales en} pos de servicios básicos. Prioritario fue ajustar las tarifas de los servicios, aumentar la transparencia de la gestión y rendición de cuentas, con el objetivo de lograr allegar recursos para agua. Un equipo encabezado por expertos colombianos ${ }^{32}$ con experiencia en la regeneración de gobernabilidad desde los municipios, logró introducir aumentos significativos en los ingresos propios de los municipios. $^{33}$

Una de las regiones de mayor impacto del programa fue el Departamento de Alta Verapaz, con municipios que vivieron un salto relevante en sus recursos. El aumento de la cantidad de contribuyentes inscritos en el padrón del Impuesto Único sobre Inmuebles (IUSI) en los municipios de Alta Verapaz, período 2007 al 2011, fue el siguiente: Carchá, $857 \%$; Tactic, 609\%; San Cristobal, 132\%; Cobán, en el padrón de usuarios del agua potable, 38\% (Promudel-GIZ, 2012).

b) Activar la participación ciudadana en la valoración del agua. Con la asistencia de expertos (as) en participación social y personal bilingüe

32 Presidido por el economista Jorge Monroy, quien otrora fuera alcalde de Usaquén, Distrito 1 de Bogotá, Colombia. 33 Colombia tuvo en la reforma fiscal de Bogotá de comienzos de los años noventa el combustible que permitió a dicha capital, Medellín y cientos de gobiernos locales más, valorar sus propias capacidades de movilizar recursos para hacer frente a la violencia, la desigualdad y la ausencia de gobierno. 
guatemalteco, conocedor de las lenguas y culturas mayas ${ }^{34}$, así como de los procesos de paz, se activaron obras cofinanciadas de agua potable con los Consejos Comunitarios de Desarrollo (Cocode) y se asesoró a los Consejos Municipales de Desarrollo (Comude) para que, mensualmente, en sus reuniones, se hiciera rendición de cuenta y se priorizaran los proyectos de agua.

El Comude, como parte del Sistema de Consejos de Desarrollo, es el medio principal de participación de los habitantes de un municipio en la gestión pública local, lo cual permite llevar a cabo el proceso de planificación del desarrollo municipal de manera democrática. En cada municipio debe funcionar un Comude, integrado del siguiente modo: ${ }^{35}$

- Alcalde Municipal, quien lo preside y coordina;

- Síndicos (revisores de cuentas) y Concejales designados por la Corporación o Concejo Municipal;

- Representantes de los Cocode, hasta un número de 20 , designados por los coordinadores de dichos Consejos;

- Representantes de las entidades públicas con presencia en el municipio;

- Representantes de las organizaciones o entidades civiles con presencia en el municipio.
Formalmente, el Comude es una instancia o espacio de consulta, asesoría y concertación en el municipio, creado para formular propuestas de solución y sugerir al Concejo Municipal su aplicación o aprobación. Funciona a partir de las propuestas hechas por los representantes de los Cocode y demás organizaciones comunitarias, para ejecutar procesos de participación e impulsar el desarrollo con visión de municipio. Normalmente, en los Comude se aprueba un listado de obras que propone el Alcalde, pero, con la intervención de Promudel, crecientemente se fueron realizando pre-Comudes para hacer una tabla de debate e incluir temas de interés de las comunidades, incluidas la revisión de los informes sobre calidad del agua, los sistemas de diagnósticos visuales (con fotografías) de los problemas municipales, donde la falta de agua y los basurales ocuparon un papel protagónico.

El trabajo de Promudel con los Cocode permitió realizar más de un centenar de obras de agua potable, donde la comunidad, el municipio y el Programa aportaban recursos y fiscalizaban las obras. Comunidades pobres aportaron lo suyo cavando zanjas, preparando terrenos y aportando jornaleros para colocar tubos y otras obras complementarias. Las sinergias positivas fueron evidentes: municipios obligados a transparentar los proveedores de insumos, comunidades que aceptan dar su contribución, y autoridades que van aprendiendo la prioridad en agua y su saneamiento.

34 Guillermo García, Sandra González, Dilia Co, entre otros, tenían dos décadas de trabajo vinculados a la cooperación internacional en zonas mayas.

35 Artículo 11 de la Ley de Consejos de Desarrollo Urbano y Rural y artículo 42 del Reglamento de Ley. 
c) Creación de unidades profesionales de servicios públicos municipales. Al inicio del Programa (2007), solo cinco de los 40 municipios tenía un responsable a cargo de una unidad técnica del agua. La mayoría tan solo contaba con un fontanero, el cual reparaba roturas de cañerías en las zonas céntricas, sin obedecer a una política de mantención, ni contar con presupuesto o una visión de los sistemas de las aldeas y comunidades. Siete años después, el 2012, al menos 28 municipios lo tenían oficializado y 24 de ellos con un nivel visible de inversión, acercándose así a la meta de lograr transformaciones medibles en el $60 \%$ de los municipios del programa.

La tarea, a cargo de la ingeniero guatemalteca Ana Victoria Rodríguez, fue educar, crear manuales y recorrer los municipios con los equipos departamentales para lograr que los alcaldes se animaran a crearan la Oficina Municipal de Servicios Públicos, entendida ésta como el conjunto de normas $y$ actividades interrelacionadas entre sí, cuya ejecución busca la prestación de los servicios bajo criterios de calidad, eficiencia, universalidad, eficacia, continuidad y economía. Estos criterios apuntan a lograr una gestión municipal moderna, que busca provocar un aumento de los ingresos, reducción de gastos y mejoramiento del rendimiento (Promudel, 2011).
La propuesta de un Modelo de Gestión de los Servicios Públicos Básicos Municipales surgió luego, con la intención de dotar a las municipalidades de herramientas que les permitieran responder con acciones administrativas al cuerpo constitucional, normativo y reglamentario del país, considerando los macroprocesos que se debían encarar: organizacional, mantenimiento de los servicios, saber cobrar y comercializar. Lo anterior implicó asumir, junto con los equipos departamentales, la ardua tarea de hacer catastros de las instalaciones, generar ordenanzas de uso y cobro, instalar medidores del consumo (contadores), entre otras, aunque hubiera que hacer frente a amenazas hasta de linchamiento de quienes se oponían, obviamente con estrategias pragmáticas de persuasión y de invitación a representante de las comunidades y municipios donde estos procesos críticos -como el clorar- si se daban, para que compartieran sus experiencias. 


\section{Cuadro n². Macroprocesos del Modelo de Servicios Públicos Municipales, Promudel.}

\begin{tabular}{|c|c|c|}
\hline $\begin{array}{l}\text { MACRO- } \\
\text { PROCESO }\end{array}$ & PROCESO & PROCEDIMIENTO \\
\hline \multirow{12}{*}{ 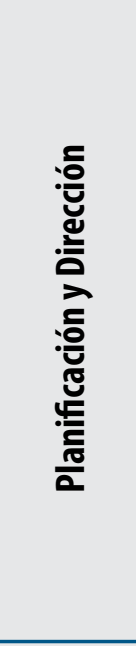 } & \multirow{4}{*}{ Organización } & Diagnóstico y línea base \\
\hline & & Definición del modelo organizacional \\
\hline & & Aprobación del modelo organizacional \\
\hline & & Adopción de manual de funciones \\
\hline & \multirow{2}{*}{$\begin{array}{l}\text { Presupuesto y } \\
\text { Financiación }\end{array}$} & Proyectar volúmen de ingresos \\
\hline & & Elaborar proyecto de presupuesto de ingresos y gastos \\
\hline & \multirow{3}{*}{$\begin{array}{l}\text { Programación de } \\
\text { obras e inversiones }\end{array}$} & Diagnóstico de necesidades de bienes e infraestructura \\
\hline & & Determinar procedencia de recursos para inversión \\
\hline & & Formular plan de compras e inversiones \\
\hline & \multirow{3}{*}{$\begin{array}{c}\text { Controly } \\
\text { seguimiento }\end{array}$} & Identificación de factores de riesgo en procesos de la Unidad \\
\hline & & Adopción de medidas correctivas \\
\hline & & Verificación de resultados \\
\hline \multirow{13}{*}{ 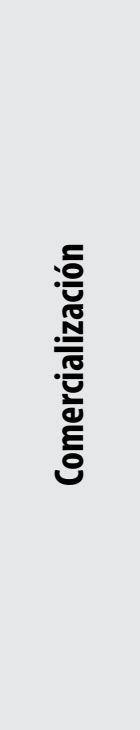 } & Tarifas & Análisis de costos y tarifas \\
\hline & Censo de usuarios & Levantamiento de Información \\
\hline & Reglmento & Elaboración o revisión del reglamento \\
\hline & \multirow{3}{*}{ Recaudación } & $\begin{array}{l}\text { Incorporación de usuarios (padrón de suscriptores por tipo de uso del } \\
\text { servicio) }\end{array}$ \\
\hline & & Definición de ruta (lectura de medidores) \\
\hline & & Facturación (generación de aviso de cobro) \\
\hline & \multirow{4}{*}{$\begin{array}{l}\text { Control del } \\
\text { NO Pago (Cartera } \\
\text { Morosa) }\end{array}$} & Registro de usuarios morosos \\
\hline & & Cobro persuasivo (acuerdos de pago) \\
\hline & & Corte de los servicios \\
\hline & & Reconexión \\
\hline & \multirow{3}{*}{ Atención al Usuario } & Solicitud de servicio \\
\hline & & Atención a quejas y reclamos \\
\hline & & Info. sobre la susp. del servicio por sit. de emergencia o coyunt. \\
\hline
\end{tabular}




\begin{tabular}{|c|c|c|}
\hline $\begin{array}{l}\text { MACRO- } \\
\text { PROCESO }\end{array}$ & PROCESO & PROCEDIMIENTO \\
\hline \multirow{12}{*}{ 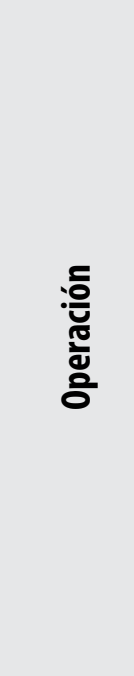 } & \multirow{2}{*}{ Suministro de Agua } & Monitores de calidad de agua \\
\hline & & Tratamiento de agua \\
\hline & \multirow{3}{*}{ Aguas Residuales } & Definición de la línea base (estudio técnico) y monitoreo \\
\hline & & Tratamiento \\
\hline & & Disposición final \\
\hline & \multirow{2}{*}{$\begin{array}{l}\text { Mantenimiento de } \\
\text { Redes }\end{array}$} & Revisión y reparación de redes de acueducto \\
\hline & & Revisión y reparación de redes de alcantarillado \\
\hline & \multirow{4}{*}{ Aseo } & Recolección y transporte \\
\hline & & Trataiento \\
\hline & & Disposición final \\
\hline & & Limpieza de espacio y bienes públicos \\
\hline & $\begin{array}{c}\text { Func. en Situaciones } \\
\text { de Emerg. }\end{array}$ & Identificar debilidades en los sistemas \\
\hline \multirow{5}{*}{ 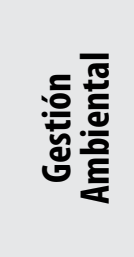 } & \multirow{3}{*}{$\begin{array}{l}\text { Protección de } \\
\text { Cuerpos de Agua }\end{array}$} & Reforestación \\
\hline & & Cerramiento (colocación de cercas) \\
\hline & & Control a disposición de agentes contaminantes \\
\hline & \multirow{2}{*}{$\begin{array}{l}\text { Gestión Int de } \\
\text { Desechos Sólidos }\end{array}$} & Campañas de separación en la fuente \\
\hline & & Reutilización de residuos orgánicos y reciclables \\
\hline
\end{tabular}

Fuente: Modelo de Gestión de Servicios Públicos, PROMUDEL,

2011. 
Uno de los buenos ejemplos gestión fue el de la Municipalidad de San Pedro Carchá, departamento de Alta Verapaz, a partir de la creación de una Oficina de Aguas Rurales. La Municipalidad había creado la Unidad de Servicios Públicos el 2008, poniendo especial énfasis en el servicio de agua. Si se toma en cuenta que este municipio tiene solo un $7 \%$ de área urbana, la responsabilidad de brindar servicios de agua en el área rural es muy grande, con el agravante que el territorio no cuenta con suficientes fuentes de agua superficiales. Con estos factores a la vista y otros más, durante el 2011, producto de la política pública de agua, la cual fue apoyada por la cooperación internacional con objetivos y metas claras en el corto, mediano y largo plazo, la Municipalidad creó la Oficina de Aguas Rurales dentro de la Unidad de Servicios Públicos. La oficina quedó integrada con 8 técnicos de campo, 1 asistente y un coordinador y se fijó como meta ampliar la cobertura y mejorar la calidad de agua a nivel de 60 comunidades en cinco años. La calidad del agua debía traducirse en la entrega de agua segura a la población, mediante la aplicación de cloro, producida por la misma Unidad, garantizando así la sostenibilidad de su producción a largo plazo.

Otro logro, contra la corriente mayoritaria que rechazaba colocar medidores para cuidar el uso del agua, fueron las cinco comunidades del Quiché, que tenían serios problemas de continuidad del servicio. Al poner contadores de servicio, lograron un suministro continuo del agua, las 24 horas del día.
Los miembros de los comités aprendieron términos nuevos, tales como: metro cúbico, dotación, mililitro, así como aprendieron a registrar el consumo de agua mensual, para luego utilizar la información y analizarla. Así lograron vencer la principal resistencia de la comunidad: el temor a que los contadores de agua significaran la privatización del servicio. Las comunidades beneficiadas con estos adelantos fueron Chicabracan I, Lemoa y Panajaxic, de Santa Cruz de El Quiché; Buena Vista de Chinique; y Chutinimit de Sacapulas.

\section{d) Políticas públicas aprobadas por el Concejo Municipal. El proceso de} persuadir a los agentes políticos de la rentabilidad de invertir en agua fue complejo y obligó a capacitar, exponer experiencias de Chile ${ }^{36}$ y Colombia, así como conocer casos positivos de Guatemala, entre ellos las empresas de agua de Ciudad de Guatemala, Quetzaltenango, Flores (Petén) y el pequeño municipio de Guastatoya, el cual tenía desde hace treinta años una oficina de aguas y drenaje (alcantarrillado) con registro de usuarios, potabilización de las aldeas y baja mortalidad infantil (Valenzuela, 2010).

La complejidad de la intervención a realizar se daba a partir de la cúpula del Municipio, con el intento de persuadir a sus autoridades. El primer paso del macroproceso de planificación y dirección implicaba su participación en el estudio cuidadoso de los servicios públicos a su cargo. Los elementos claves a contemplar en este

36 En particular, el Sistema de Agua Potable Rural (APR), como expresión de la coordinación entre el nivel comunitario con el regional, por la vía de los proyectos de apoyo en infraestructura y gestión que el Ministerio de Obras Públicas pacta con empresas sanitarias. 
diagnóstico fueron: organización administrativa, presupuestación y financiamiento, programación de inversión, mantenimiento, seguimiento y control. En síntesis, planificar y dirigir es pensar permanentemente en una realidad y prever sus potenciales cambios. El foco estaba puesto en los dividendos políticos y sociales de ampliación de cobertura y mejoramiento de la potabilización del agua. Factor clave fue contar con un líder maya, Valentín Tavico, quien había sido secretario ejecutivo de la Asociación Guatemalteca de Alcaldes y Autoridades Indígenas (AGAAI) durante una década, con experiencia en cooperación e interculturalidad. Es decir, con capacidad de entender tanto los códigos culturales como políticos de los alcaldes, autoridades mayas e integrante de los concejos en subetnias diferentes: Mam de Huehuetenango, Achís de Baja Verapaz, Quebchís de Alta Verapaz, Kichés, Sacapultecos e Ichiles en el Kiché, entre otros. Tavico coincidió en que había poca esperanza en los sistemas estatales: ausencia del Ministerio de Salud en agua potable, de la Contraloría/Procuraduría en sancionar, además de la cooptación de los fondos departamentales para proyectos mediocres, sin proyección o abiertamente superfluos. Por tanto, el acento estaría en lograr focalizar los municipios hacia el cumplimiento de los Objetivos del Milenio (menos pobreza, más salud).

Por su parte, el Gobierno de Alvaro Colom (2008 - 2012) implementó programas de ayuda social monetaria condicionada y en alimentos, a las familias vulnerables -en sintonía con el sistema Chile Solidario o el brasileño Fome Zero, de lucha contra el hambre-, pero descuidó la inversión en infraestructura, por lo cual era pro- picio pedir a los municipios un mayor protagonismo en el tema del agua. De esta manera, se recorrieron los cuarenta municipios debatiendo los Objetivos del Milenio y el aporte municipal susceptible de llevar a cabo, para conseguir agua potable que permitiera, a su vez, reducir enfermedades importantes. La intervención realizada ha sido operacionalizada en un esquema con sus pasos prácticos, el que se verá a continuación.

Fases básicas para una política municipal de agua: el caso de Guatemala (Valenzuela, 2010)

\section{Difusión de políticas públicas (PP) en tomadores de decisión}

Los líderes deben comprender que la Política Pública es específica, no es el Plan general del Municipio, pero tampoco un proyecto más. La metodología básica debe comprenderse. La totalidad del Concejo, los principales funcionarios, líderes del Comude y líderes de opinión deben quedar armonizados en los conceptos.

\section{Diagnóstico del problema prin-} cipal sobre el cual se puede actuar (indicadores claros)

Compartir a nivel político local y de los líderes de opinión y comunitarios, cuál es el problema principal sobre el cuál formular una política pública. Debe ser muy concreto y aterrizado a lo que se tiene. No se puede hacer una PP sobre el precio internacional del café porque no se controlan esas variables ni se tiene la competencia necesaria. En cambio, si se puede actuar en agua, basura, fomento productivo local, alfabetización de adultos, entre otros. Debe haber indicador-meta-propósito claro. 


\section{Amplio debate en Comisión Plural político-social}

Los ingleses hablan de que más importante que una ley es su debate previo (greenpaper) y su implementación (que se lleve a la práctica y se cumpla). Por eso, no hay que temer el debate y la discusión amplia. El modelo que más se utiliza es crear una amplia comisión, con mandato claro, que debate un tiempo determinado (noventa días, por ejemplo) sobre una política pública y emita sus recomendaciones al cuerpo político (nacional o municipal).

\section{Aprender de las mejores expe- riencias cercanas}

En Política Pública hay que tener humildad para reconocer qué no se sabe y aprender a encontrar soluciones buenas, pragmáticas y sustentables en el tiempo. Por ejemplo, no inventar un sistema de incineración de la basura en un país tropical con basura con agua, ni construir sistemas de transporte vía metro en países con crisis energética, ni generar sistemas de apoyo a los más pobres si estos no son universales (para evitar la discrecionalidad). Por el contrario, hay que ir a mirar aquello que funciona y que es cercano.

\section{Diseño y Formulación de la Políti- ca Pública}

Con buena asesoría profesional y político-técnica se diseña y formula la política pública. Esta debe contener, al menos: (a) Objetivo, (b) Meta e indicador a modificar, (c) Plan de acciones, proyectos y responsables, (d) Financiamiento, (e) Reglamentos, leyes o acuerdos, (f) Etapas y su temporalidad (plazos) y (g) Consejo Plural de Vigilancia o monitoreo.
6. Pacto social, político y financiero para realizar la política pública

Tras fijar el área, debatir diagnóstico y enfoques, corresponde observar experiencias exitosas en torno de situaciones similares. Para diseñar y formular la Política Pública, además, debe existir un pacto social entre los actores políticos, con poder y comunitarios, para su implementación. Esto implica voluntad política, corresponsabilidad y aportes a la financiación.

Ejemplos: (1) En torno a una política ambiental, deben estar presente las empresas que contaminan y tener plazas para colocar filtros. (2) Mejorar la calidad del agua local implicará acciones municipales de cloración, mantenimiento y acuerdo con la comunidad para que ésta pague lo que cuesta el servicio mejorado.

\section{Implementación coherente y con calidad}

La implementación debe hacerse con excelencia, calidad y transparencia. Tener presente lo que se denomina globalmente empresa o unidad ideal de servicio. Es decir, aspirar al nivel organizacional de excelencia para hacer el proyecto, evitando el despilfarro que acarrean los costos adicionales. Por ejemplo, hay que evaluar el costo-beneficio de comprar camiones versus contratar por horas un servicio. Averiguar si es mejor sacar el agua de una napa subterránea, aunque implique pagar mil dólares de electricidad al mes, que traer el agua de un nacimiento a $20 \mathrm{kms}$. de distancia, sin tener el personal necesario para vigilar y reparar. $\mathrm{O}$ al revés, si la electricidad es tan cara, a lo mejor vale la pena acarrear el agua desde una distancia prudente. 


\section{Monitoreo, evaluación y rediseño}

Toda política pública implica una actividad de monitoreo, vigilancia y no tener miedo a la crítica y evaluaciones. Eso permite rediseñar y colocar correctivos a tiempo.

Las buenas experiencias permiten que existan consejos o directorios de súper vigilancia neutrales, pluralistas y profesionales, que emitan recomendaciones a alto nivel. En cada municipio ello es posible. Comisiones de los Comude y personalidades reconocidas de la comunidad, son ideales para este ejercicio.

\section{Política Pública Agua Potable para Todos del Municipio de Cobán}

Entre los ejemplos de mayor sustentabilidad, destaca el Municipio de Cobán, de 200 mil habitantes, el cual formuló política, aumentó recursos, creó servicio e invirtió con eficacia para la obtención de agua segura.

Las claves de su política pública fueron las siguientes:

- Plan maestro para cobertura de agua y drenaje.

- Inversión en proyectos específicos e ingeniería.

- Aumento de la tarifa a rangos sustentables.

- Tarifa diferenciada para el consumo doméstico e industrial.

- Creación de Unidad de Servicios de Agua.

- Adecuada licitación y administración frente a donantes nacionales e internacionales.

- Instalación de medidores-contadores y proyección de plantas de tratamiento.

- Plan de inversión en las aldeas y sistemas rurales.

- Carpeta de proyectos presentados a diversas fuentes nacionales, departamentales y de cooperación internacional.

1. Valenzuela, Esteban. 2010. Guía de Políticas Públicas Municipales. Guatemala, Promudel. P 31. 
e) Monitoreo nacional y difusión de innovaciones

El quinto componente fue monitorear a los municipios con un Ranking que comenzó siendo para los cuatro departamentos, pero que, debido al éxito del instrumento creado por Jesús Coronado, Segeplan lo convirtió el año 2012 en un Ranking Nacional, destinado a medir a los 330 municipios guatemaltecos. El efecto de competencia de gestión entre los buenos municipios ayudó a que se les hicieran preguntas a los Comunde en relación con sus gestiones de agua peor evaluadas. A los municipios más exitosos se les premió con viajes para conocer buenas experiencias en Colombia y en la propia Guatemala.
De manera complementaria, se realizaron ferias en los departamentos con las mejores experiencias y seminarios en la Ciudad de Guatemala. Además, se editaron $C D$ y libros, con el objeto de estimular la replicabilidad y el up scaling ${ }^{37}$, buscando que los entes nacionales promocionen los instrumentos.

En cada Consejo Departamental de Desarrollo (Codede), se divulgó el ranking y se explicaron las razones para incluir los 23 indicadores que, durante cinco años, se indagaron en cada municipio.

\section{Cuadro n³. Indicadores del Ranking de Medición de Gestión municipal de PROMUDEL}

\section{a. Indicadores de Participación Ciudadana}

1. Asistencias a reuniones de Comude de concejales acreditados. Es obligación de las corporaciones municipales designar a por lo menos dos de sus miembros, aparte del Alcalde Municipal, para su representación en el Comude. En este indicador se mide tanto la designación existente como las asistencias reales de los representantes responsables. Será verificada en la municipalidad la existencia de Acuerdos Municipales, en los que debe constar la designación y en las actas del Comude se verifica la asistencia de los designados en las reuniones de cada mes.

2. Número de reuniones de Comude realizadas en el año.

Es el número total de reuniones que el Comude ha realizado, verificando el cumplimiento de la obligación de realizarlas mensualmente. Se verifica la existencia de las actas del Comude.

3. Representación en el Comude de grupos de mujeres, jóvenes e indígenas. La representación de la sociedad civil organizada se mide, específicamente, mediante la presencia de grupos de mujeres, jóvenes e indígenas. Se asigna un punto por cada grupo que esté representado en el Comude del municipio. 
4. Asistencia del Alcalde a las reuniones del Comude.

El Alcalde Municipal preside el organismo. En este indicador se mide la cantidad de reuniones del Comude en las que el Alcalde se hace presente.

5. Representación territorial del Comude. Se refiere a la acreditación de los representantes de los Cocode de segundo nivel (en el caso de municipalidades en las que haya microregionalización, y, en los casos en que no, se verifica la acreditación de Cocode de primer nivel). Se contrasta este número frente a la cantidad de aldeas, caseríos, microrregiones, zonas, etc.

\section{Funcionamiento del Comude.}

En este indicador se miden varios aspectos: $1^{\circ}$, acaso se definió un cronograma de reuniones al inicio de año; $2^{\circ}$, en qué proporción se cumplió este cronograma; $3^{\circ}$, en caso de haberlo definido, verificar si se definió, presentó y aprobó una agenda al inicio de las reuniones; $y, 4^{\circ}$, la existencia de comisiones y si éstas tienen plan de trabajo definido. También se constata la existencia de comisiones de Derechos Humanos, Transparencia, Servicios Públicos, Participación Ciudadana, Finanzas y Mujeres.

7. Existencia de reglamento interno del Comude.

Se verifica acaso existe un reglamento interno del organismo, en cada una de las municipalidades en las que se realiza la medición.

8. Temas relevantes tratados en el Comude.

Este indicador busca establecer si el organismo es un espacio de deliberación en el que se abordan los temas más relevantes para la población. Se verifica en sus actas, acaso en las reuniones se incluyeron temas relacionados con los servicios públicos (suministro de agua potable, aguas servidas, tratamiento de desechos sólidos), temas ambientales, desarrollo económico local y gestión de riesgo.

\section{b. Indicadores de Efectividad Gubernamental}

9. Cobertura de agua área urbana.

Se verifica en el Padrón de agua del Sistema Integrado de Administración Financiera y Control (SIAF) el número de conexiones registradas y se contrasta con el número de hogares urbanos, definido usando las proyecciones de población del censo de 2002.

10. Cobertura recolección de Basura Área Urbana.

Se verifica en el Padrón de arbitrios del SIAF el número de conexiones registradas y se contrasta con el número de hogares urbanos, definido usando las proyecciones de población del censo de 2002. 
11. Elementos de Servicio al Ciudadano.

En este indicador se refleja la existencia o no de elementos de servicio que ayudan a mejorar la relación del vecino con la municipalidad. En el trabajo de campo se verifica: $1^{\circ}$, existencia o no de un puesto de información (con la función de orientación); $2^{\circ}$, rotulación de oficinas en español, idioma maya y/o un sistema de colores para ayudar a ubicar a vecinos analfabetos; $3^{\circ}$, existencia del directorio de trámites; $4^{\circ}$, existencia del manual de trámites; $5^{\circ}$, existencia de una política de Servicio al Ciudadano; y $6^{\circ}$, existencia o no de un compromiso de atención elaborado por los funcionarios municipales, publicado en un lugar visible.

\section{Ingresos propios per capita.}

Las fuentes de información para la construcción de este indicador son las proyecciones de población del censo 2002 y el SIAF. Se contrasta el monto total de los ingresos propios contra el total de la población proyectada para el año analizado.

\section{Independencia financiera.}

Este indicador mide el monto que representan los ingresos propios dentro del total de ingresos quitando la deuda.

14. Existencia de políticas públicas o líneas estratégicas de acción.

Se verificó si en la municipalidad existen documentos en los que se defina una línea estratégica o una política pública. Se incluye dentro de los documentos verificados el Plan de Desarrollo Municipal.

15. Porcentaje del presupuesto destinado a líneas estratégicas.

Si el municipio tiene líneas estratégicas o políticas públicas definidas, se verifica el monto destinado a ellas en la ejecución presupuestaria de egresos del año.

\section{Planeación operativa y ejecución.}

Se verifica la existencia de un plan operativo para el año y se compara el número de proyectos definido inicialmente contra el que efectivamente ha sido ejecutado en el año o se encuentra en ejecución.

\section{Cumplimiento Plan de Desarrollo Municipal.}

En los planes de desarrollo se define un conjunto de proyectos que debe realizarse especificando el año en el que se pretende realizarlos. En este indicador se contrasta el número de proyectos definido para ejecutar en el año y el número que efectivamente se ha ejecutado o se encuentra en ejecución.

\section{c. Indicadores de Transparencia}

18. Número de rendiciones de cuentas trimestrales en el año.

Se verifica en las actas del Comude la rendición de cuentas, de acuerdo a lo establecido en el Código Municipal. La obligación, para el año 2009, era la realización de una rendición de cuentas trimestral. 
19. Existencia de la Unidad de Información Pública.

La Ley de Acceso a la Información Pública (LAIP) exige a todos los sujetos obligados, incluyendo las municipalidades, que creen una Unidad de Información Pública. Se verifica la creación de la Unidad mediante acuerdo municipal.

20. Porcentaje de Información Pública de Oficio disponible.

En el artículo 10 de la LAIP se especifica un conjunto de documentos que la municipalidad debe tener disponibles para consulta inmediata en todo momento, ya sea de forma física o en una página web. Se verifica en cada municipalidad acaso esa información está disponible en los términos que especifica la ley.

21. Presentación informe de ejecución presupuestaria.

Las Municipalidades deben presentar en el Comude, durante el primer trimestre del año siguiente, un informe de la ejecución presupuestaria del año anterior. En el primer trimestre de 2010, cada municipalidad debió presentar en el Comude su informe y esto es lo que puede constatarse en este indicador.

22. Información presupuestaria disponible en medios diversos.

Una buena práctica para la transparencia es la de mantener informada permanentemente a la población a través de diversos medios y no solo hacerlo trimestralmente como exige la ley. En este indicador se reconoce el esfuerzo de las municipalidades en hacer trifoliares, cartillas, carteleras, spots radiales, programas de televisión, etc., en los que se presente información presupuestaria y el estado de avance de los proyectos comprometidos.

23. Monto asignado en Guatecompras como porcentaje de egresos.

En este indicador se contrasta el monto de las compras de la municipalidad que se encuentra registrado en la página web del sistema Guatecompras, comparado con el monto total de compras realzadas, para verificar qué porcentaje de la ejecución presupuestaria ha sido realizada por esta vía.

Jesús Coronado. 2011. Sistema de Medición de Indicadores de Gestión Municipal. Promudel. 


\section{III.2. El impacto del Programa}

Tras cinco años de ejecución de Promudel, los municipios cambiaron, mejoraron y perfeccionaron su gestión del agua, hasta en un $70 \%$, tal como se muestra en los siguientes cuadros con los resultados de las mediciones realizadas en los períodos que se indican:

Cuadro $\mathrm{n}^{\circ} 4$. Tabla comparativa de porcentaje de gasto en agua por departamento $y$ municipios con más de un $10 \%$ de inversión en agua.

\begin{tabular}{|l|c|c|c|c|} 
& $\begin{array}{c}2008 \\
\text { Número de } \\
\text { Municipios que } \\
\text { invierten más de } \\
\text { un } 10 \%\end{array}$ & $\begin{array}{c}2008 \\
\% \text { gasto global } \\
\text { en agua como } \% \\
\text { gasto municipal } \\
\text { total }\end{array}$ & $\begin{array}{c}2011 / 12 \\
\text { Número de } \\
\text { Municipios que } \\
\text { invierten más de } \\
\text { un } 10 \%\end{array}$ & $\begin{array}{c}2011 / 12 \\
\% \text { gasto global } \\
\text { en agua como } \%\end{array}$ \\
$\begin{array}{l}\text { Kiché } \\
\text { gasto municipal } \\
\text { total }\end{array}$ \\
\hline Alta Verapaz & 5 & $12 \%$ & 8 & $19 \%$ \\
\hline Baja Verapaz & 4 & $12 \%$ & 9 & $18 \%$ \\
\hline Huehuetenango & 5 & $10 \%$ & 5 & $25 \%$ \\
\hline $\begin{array}{l}\text { TOTAL } \\
\text { 4 Departamentos }\end{array}$ & 20 & $11 \%$ & 8 & $15 \%$ \\
\hline
\end{tabular}

Fuente: Progress Report PROMUDEL 2012. GIZ-Guatemala.p.3

Cuadro $n^{\circ} 5$. Porcentaje de la inversión total municipal destinado al ciclo del agua*, municipios Departamento Quiché, 2008-2011.

\begin{tabular}{|l|c|c|}
\multicolumn{1}{r}{ Municipios Departamento de Quiché } & $\mathbf{2 0 0 8}$ & $\mathbf{2 0 1 1}$ \\
\hline Santa Cruz del Quiché & $12 \%$ & $35 \%$ \\
\hline Chinique & $15 \%$ & $10 \%$ \\
\hline Chichicastenango & $11 \%$ & $18 \%$ \\
\hline Patzité & $7 \%$ & $2 \%$ \\
\hline San Antonio Llotenango & $8 \%$ & $11 \%$ \\
\hline Cunén & $7 \%$ & $9 \%$ \\
\hline Sacapulas & $19 \%$ & $30 \%$ \\
\hline Canillá & $2 \%$ & $21 \%$ \\
\hline Uspantán & $15 \%$ & $10 \%$ \\
\hline Sabcabajá & $12 \%$ & $8 \%$ \\
\hline Porcentaje Departamental & $12 \%$ & $19 \%$ \\
\hline
\end{tabular}

* Incluye gastos de inversión, personal, mantenimiento e insumos.

Fuente: Elaborado por Paola Sholstak, con supervisión de EV en base a información oficial de los Municipios. 
Cuadro n6. Porcentaje de la inversión total municipal destinado al ciclo del agua*, municipios Departamento de Alta Verapaz, 2008-2011.

\begin{tabular}{|c|c|c|}
\hline Municipios Departamento de Alta Verapaz & 2008 & 2011 \\
\hline Cobán & $13 \%$ & $20 \%$ \\
\hline San Pedro Carchá & $17 \%$ & $22 \%$ \\
\hline Chisec & $5 \%$ & $15 \%$ \\
\hline Tactic & $9 \%$ & $27 \%$ \\
\hline San Juan Chamelco & $12 \%$ & $3 \%$ \\
\hline San Cristóbal Verapaz & $15 \%$ & $28 \%$ \\
\hline Raxruhá & $13 \%$ & $13 \%$ \\
\hline Santa Cruz Verapaz & $10 \%$ & $30 \%$ \\
\hline Chahal & $9 \%$ & $16 \%$ \\
\hline Fray Bartolomé de las Casas & $8 \%$ & $27 \%$ \\
\hline Porcentaje Departamental & $12 \%$ & $19 \%$ \\
\hline
\end{tabular}

* Incluye gastos de inversión, personal, mantenimiento e insumos.

Fuente: Elaborado por Paola Sholstak, con supervisión de EV en base a información oficial de los Municipios.

Cuadro $\mathrm{n}^{\circ}$ 7. Porcentaje de la inversión total municipal destinado al ciclo del agua*, municipios Departamento de Baja Verapaz, 2008-2011.

\begin{tabular}{|l|c|c|}
\multicolumn{1}{|c}{ Municipios Departamento de Baja Verapaz } & $\mathbf{2 0 0 8}$ & $\mathbf{2 0 1 1}$ \\
\hline El Chol & $8 \%$ & $53 \%$ \\
\hline Granados & $0,8 \%$ & $0 \%$ \\
\hline Purulhá & $22 \%$ & $30 \%$ \\
\hline Rabinal & $15 \%$ & $10 \%$ \\
\hline Salamá & $13 \%$ & $18 \%$ \\
\hline San Jerónimo & $5 \%$ & $4 \%$ \\
\hline San Miguel Chicaj & $1 \%$ & $5 \%$ \\
\hline Cubulco & $34 \%$ & $9 \%$ \\
\hline Porcentaje Departamental & $10 \%$ & $32 \%$ \\
\hline
\end{tabular}

*Incluye gastos de inversión, personal, mantenimiento e insumos.

Fuente: Elaborado por Paola Sholstak, con supervisión de EV en base a información oficial de los Municipios. 
Cuadro ${ }^{\circ}$ 8. Porcentaje de la inversión total municipal destinado al ciclo del agua*, municipios Departamento de Huehuetenango, 2008-2011.

\begin{tabular}{|l|c|c|}
\hline \multicolumn{1}{|c|}{ Municipios Departamento de Huehuetenango } & $\mathbf{2 0 0 8}$ & $\mathbf{2 0 1 1}$ \\
\hline Chiantla & $35 \%$ & $16 \%$ \\
\hline Malacatancito & $10 \%$ & $10 \%$ \\
\hline Santa Bárbara & $9 \%$ & $10 \%$ \\
\hline Santa Eulalia & $0,2 \%$ & $10 \%$ \\
\hline San Antonio Huista & $7 \%$ & $23 \%$ \\
\hline San Pedro Necta & $13 \%$ & $8 \%$ \\
\hline Todos Santos & $6 \%$ & $8 \%$ \\
\hline San Miguel Acatán & $1 \%$ & $13 \%$ \\
\hline San Mateo Ixtatán & $2 \%$ & $6 \%$ \\
\hline Unión Cantinil & $5 \%$ & $12 \%$ \\
\hline Huehuetenango & $10 \%$ & $25 \%$ \\
\hline Porcentaje Departamental & $11 \%$ & $14 \%$ \\
\hline
\end{tabular}

*Incluye gastos de inversión, personal, mantenimiento e insumos.

Fuente: Elaborado por Paola Sholstak, con supervisión de EV en base a información oficial de los Municipios.

IV. SUGERENCIAS PARA HACER EFICAZ UN SISTEMA DE GOBERNABILIDAD DEL AGUA EN GUATEMALA

Promudel fue un programa de siete años, que contó con una alta inversión de dos países donantes (Alemania y Suecia), además de haber movilizado equipos profesionales y recursos de Guatemala, y contado con la colaboración de entidades y profesionales de Chile y Colombia. Pero, fue esencialmente la voluntad de los equipos municipales de Guatemala, del mundo maya, el hecho determinante a la hora de introducir cambios y nuevas prácticas en sus municipios. No obstante la valorización de dichas experiencias, se hace evidente la necesidad de recoger las recomendaciones de estudios especializados para mejorar la gobernabilidad del agua, promover la corresponsabilidad y buscar un Estado pro activo y no ausente en el tema como el actual.
El agua no se cuida ni se provee por la mera dictación de leyes y normas. Se necesitan programas de apoyo, entes reguladores, sanciones reales a los que fallan por negligencia. Se requiere, por lo tanto, gobernabilidad estatal sobre el agua (acción eficaz del Estado y municipios), complementado con una buena gobernanza de empresas y comunidades, en un sistema regido por reglas claras, cooperación y corresponsabilidad.

Dos investigadores de CEPAL, M. Solanes y A. Jouravlev, propusieron un rol activo del Estado en la regulación de los sistemas y alejarse de la administración directa, que lo hace perder legitimidad por los problemas de corrupción e ineficacia para proveer el servicio. Es decir, ellos promueven un enfoque pragmático y no ideologizado, donde el Estado vela por la buena calidad en forma activa, apoya los sistemas en las zonas menos ren- 
tables, y a la vez se abre a establecer modelos mixtos, ya sea de empresas municipales, privados concesionarios, servicios municipales, regionales $\mathrm{u}$ otros. El Estado debe hacer bien su rol aunque "la formulación de las políticas de agua han sido tomadas en un contexto de debilidad de las instituciones y problemas con el financiamiento para cumplir adecuadamente sus tareas" (Solanes y Juravlev, 2006:22). ${ }^{38}$

En su documento, los investigadores proponen los elementos que hacen un buen modelo de gobernabilidad, que se sintetizan a continuación:

a) Dominio público del agua, pero con derechos de uso claros, privilegiando a la gente cercana a las fuentes.

b) Estructura institucional de manejo del agua clara y diferenciada. Una institución supervisa (superintendencias o salud) y otra administra las concesiones públicas o privadas, tanto para uso de agua potable, agrícola o industrial-minero (valora la Comisión Nacional de Aguas de México, la Unidad de Agua del Ministerio de Medio Ambiente en Colombia o la Dirección de Aguas del Ministerio de Obras Públicas de Chile).

c) Combinar racionalidad económica con demandas sociales. Lograr que el sistema cobre el precio a los usuarios que permita financiar los servicios, pero considerar subsidios parciales a los sectores más pobres para no afectar su consumo. d) Buena regulación de los servicios. Aquí está el rol clave del Estado en normar y supervisar las obras, la calidad de los sistemas, la debida cloración, los sistemas de saneamiento.

e) Asignar roles adecuados a los distintos niveles de gobierno. Si hay administración privada (empresa o comunitaria) o municipal, normalmente el nivel intermedio (departamento, región) vela por apoyar en infraestructura y gestión al sector rural y comunidades pobres.

f) Participación pública. Los sistemas buscan la participación de representantes de las comunidades y ciudadanos en los consejos que fijan tarifas, políticas y reciben informes de calidad del agua.

g) Consideraciones ambientales. Se debe considerar la sustentabilidad, el manejo de cuencas, el tratamiento del agua, la evaluación rigurosa de los megaproyectos eléctricos y mineros.

h) Derechos de minorías indígenas y consumidores. No pueden soslayarse los intereses de las comunidades originarias (brindar protección y compensaciones), así como los informes de calidad y costos transparentes a los consumidores.

i) Resolución adecuada de conflictos. Se necesitan comisiones independientes y técnicas que fallen con justicia los conflictos de aguas, buscando establecer la adecuada mediación y acuerdo de actores. 
j) Toma de decisiones justa y eficaz. El marco de gobernabilidad debe permitir la toma de decisiones fluida, incluyendo períodos críticos, como en las sequías, donde suele haber serios conflictos de intereses entre zonas y sectores (hidroeléctricas, agricultura, minería o comunidades). El ente con poder resolutorio debe buscar el bien común y estar libre de la cooptación y corrupción por intereses corporativos de cualquier índole.

Guatemala tiene buenas normas que prohíben la contaminación del agua potable y que fijan los niveles aceptables de descargas de aguas residuales, pero hay omisiones en la implementación. Hace falta contar con una superintendencia de servicios sanitarios o unidad a cargo del Ministerio de Salud, un fondo adicional que apoye con recursos para agua y saneamiento a los departamentos con peores indicadores de acceso a agua y mortalidad, equipos técnicos profesionales en los departamento que apoyen a los municipios, exigir a las municipalidades perfiles básicos en los cargos de sus oficinas de servicios públicos y agua, un esquema tarifario básico nacional con elementos redistributivos, hacer obligatorio el control del consumo (medidores) y de la cloración.

Así como se hace eficientemente con la ley del tabaco, se requiere instaurar control y sanciones reales para quienes incumplen las normas básicas en agua.
Al respecto, se han dado pasos en los debates del Gabinete del Agua y se han formulado proposiciones, que acá complementamos con los caminos que pudieran adoptarse:

1. Evaluar y diferenciar las normas de calidad del agua potable, administración de drenajes e implementación progresiva del tratamiento de aguas residuales. En la experiencia comparada, se suele tener estándares distintos por zonas, por actividades, por ser un condominio urbano o una pequeña aldea.

2. Unidad Fiscalizadora: Creación de la Superintendencia de Servicios Sanitarios (agua, drenaje, tratamiento) o creación de la Unidad Nacional de Saneamiento con potestad regulatoria, fiscalizadora y sancionatoria en el Ministerio de Salud.

3. Recursos específicos: Creación del Fondo Nacional de Promoción del Agua Segura (PRO-AGUA) que de manera complementaria al financiamiento que proveen los fondos departamentales de los Codedes, se concentre en ampliar las coberturas de agua potable, drenaje y saneamiento en los departamentos que muestran los mayores déficits en acceso al agua segura, mayor prevalencia de enfermedades y mortalidad infantil.

4. Calidad de proyectos y profesionalización: se sugiere la creación del equipo profesional a cargo de los Bancos de Proyectos de Agua por Departamento, con cinco ingenieros sanitarios como mínimo. Otro modelo, es que el INFOM retome su función activa en este ámbito, o que se fusionen en una nueva delegación efectiva en los territorios los profesionales 
que se harán cargo, con independencia, de la calidad de los proyectos.

5. Transparencia y calidad de ejecutores: Se suele lograr un Sistema Nacional de Licitación de proyectos de Agua con auditorías de implementación independientes (empresas autónomas con seriedad). El registro de empresas especializadas, con solidez técnica y financiera, es deseable como requisito básico.

6. Ley de sanciones y multas a quienes incumplan las normas. Incluir montos, períodos para corregir y destitución por "notable abandono de deberes" a las autoridades públicas y municipales que omitan su implementación. La Unidad de Saneamiento del Ministerio de Salud podrá, en caso de incumplimiento grave, decretar el cierre temporal de industrias contaminantes de cursos de agua si no hacen tratamiento.

7. Programa de Agua Potable Rural (APR) por Departamento, que asesore y apoye a los municipios en su trabajo con los sistemas de agua comunitarios de aldeas y caseríos en su gestión, medición de contaminación y reposición de infraestructura para garantizar la sostenibilidad. El Estado central, en las experiencias latinoamericanas, o los gobiernos regionales autónomos, apoyan y acompañan a los municipios en brindar asistencia a las comunidades pobres y apartadas.

8. Medir calidad: Se sugiere institucionalizar un Programa trimestral de medición de calidad de todos los sistemas de agua del país, a cargo del Ministerio de Salud, lo que en parte ya se hace bien en muchos departamentos, pero no en todos. Para los sistemas privados existirá un regla- mento con la tarifa a pagar al Ministerio y/o Municipio que haga la medición. Cada delegación departamental de Salud debiera implementarlo.

9. Financiamiento: Ya sea por ley, decreto o directiva nacional, se debe dejar explícita las obligaciones del financiamiento de los sistemas de agua, el deber de financiar la operación de los sistemas municipales, incluir la instalación de medidores, y la tarifa diferenciada, que permita subsidiar parcialmente las familias más pobres. La entrega del boleto de pago del agua podría ser un requisito para ciertos trámites públicos, lo que fue muy útil con el Boleto de Aseo y Ornato, el cual, al ser obligatorio para trámites financieros, incentiva a la ciudadanía a pagarlo.

10. Inclusión del agua en una reforma tributaria. Igualmente, para financiar el sistema PRO-AGUA en los departamentos rezagados, se aconseja el financiamiento vía reforma tributaria, ya sea alzando en un punto el impuesto al consumo (IVA-AGUA) y/o el de rentas personales o de empresas (Agua para todos), especialmente las mineras y extractivas.

11. El Ministerio de Medio Ambiente (MARM), debiera ejecutar su rol de coordinador de los Consejos de Manejo de Cuencas o Microcuencas, para lo cual debiera existir un Fondo Nacional de Proyectos a los cuales anualmente las organizaciones comunitarias y mancomunidades de municipios puedan postular, a fin de implementar acciones de manejo de las mismas. Dictar normas de pago de servicios ambientales a empresas que usan recursos hídricos para reinvertirlos en dicho fondo. 
12. Los Municipios deberán especializar en forma obligatoria la gestión del agua, pudiendo crear una empresa municipal o una Unidad de Servicio de Agua, la que deberá ser conducida por un profesional con título universitario en las municipalidades con más de cincuenta mil habitantes y al menos título técnico, en aquellas con menos de cincuenta mil.

13. Control social y participación en el agua: El sistema de administración municipal concentrará en el ítem presupuestario 'agua', todos los gastos de personal, proyectos, inversión, reposición, mantenimiento, medición, supervisión de los sistemas de agua en su territorio. Además, será obligatorio dar cuenta semestralmente de la administración y calidad de los sistemas de agua al Comude respectivo. El representante del Ministerio de Salud deberá concurrir de manera obligatoria a dicha reunión.

14. Programa de apoyo a la legalización de la propiedad comunitaria y municipal del agua. Uno de los programas necesarios de apoyo es lograr la regularización de la propiedad comunitaria y municipal de nacimientos de agua, lo que requiere un acuerdo pragmático entre la valoración del agua como propiedad pública del Estado y la compensación a privados, a precios moderados, para evitar un debate ideológico que cierra las puertas al avance en esta materia.

\section{CONCLUSIÓN}

Varios observadores externos observan con curiosidad que los guatemaltecos son más estrictos que algunos países europeos en el control de las leyes anti-tabacos, siendo imposible fumar incluso en los patios de comedores de perdidos pueblos en el altiplano. Sin embargo, es el mismo país de las altísimas tasas de enfermedades estomacales y mortalidad infantil que no tiene una autoridad que controle, ayude y sancione de manera efectiva a los infractores por el derecho humano al agua segura.

Por otra parte, Guatemala ha dado pasos positivos en aumentar la cobertura de la Educación, pero sigue con rezagos significativos en la calidad de los servicios municipales, tal como hemos analizado, en especial los concernientes a todos los pasos del ciclo del agua.

La buena descentralización es también responsabilidad de las elites, el modelo de Estado, la calidad de los controles, la voluntad de hacer solidaridad estructural y no robar.

Cabe insistir en el elemento esencial del desarrollo: confianza mutua. Ella se construye pagando impuestos, con servicios de calidad y oficinas profesionales, con recursos extras para zonas pobres, controles independientes, gobernanza de calidad a nivel territorial.

Este proceso es posible de conseguir en Guatemala: requiere pacto político y concertación de una nueva agenda de reformas efectivas a nivel territorial. La mejora de los servicios no llega por prototipos ni consultorías tecnocráticas; será el resultado de un 
nuevo diseño económico e institucional del nivel local, que promueva un nuevo juego de buenas prácticas, como aquí se ha esbozado para la gobernanza del agua. La experiencia de PROMUDEL y el esfuerzo y resultados obtenidos por 25 municipios a su alero, son testimonio de que es posible lograrlo, a pesar del contexto adverso.

\section{REFERENCIAS}

Carías, D. (2011). Estudios de las Reformas Tributarias recientes en Centro América. Guatemala: GIZ-Instituto Centroamericano de Estudios Fiscales.

Cepal - GTZ. (2011). Servicios de Agua Potable y Saneamiento en Guatemala. 2011. Informe Cepal-GTZ. Santiago de Chile: Cepal-GTZ.

Espitia, G. (2008). Modelo propuesto para la Gestión de los Servicios Públicos Básicos Municipales (Acueducto, Drenaje y Recolección de Desechos Sólidos). Guatemala: Promudel.

ILPES. (2011). Conclusiones. Seminario Indicadores Tributarios. Santiago: Cepal.

Lentini, E. (2010). Servicios de Agua Potable y Saneamiento en Guatemala: beneficios potenciales y determinantes de éxito. Santiago de Chile: Cepal-GIZ.

Renner, Y. \& Hesselbarth, S. (2003) Organización de Tarifas en la Gestión Urbana del Agua. Berlín: KFW.

OPS. (2012). Situación de la DesarroIlo de las Américas, Indicadores Básicos, NY: OPS.

Pérez, JF. y Oliva, B. (2008). La conta- minación del agua y su impacto en la salud en Guatemala. Tesis de grado. Guatemala: Universidad de San Carlos.

PNUD (2012). Informe Mundial de Desarrollo Humano. New York: ONU.

PNUD-Guatemala (2011): Hacia un Estado para el Desarrollo Humano. Guatemala: PNUD - UE.

Programa Estado de la Nación (2011). Informe de la Región Centroamericana. San José de Costa Rica: CONARE.

Promudel. (2011). Manual de Servicios Públicos. Guatemala.

Promudel-GIZ (2010, 2011,2012). Progress Reports. Guatemala: GIZ.

Rodríguez, A. (2011). Gestión de los Servicios Públicos Municipales, Guatemala: Promudel-GTZ.

Rosales, M. (2012). Descentralización del Estado y Finanzas Municipales en América Latina. Santiago: FLACMA.

Segeplan (2006). Encuesta Nacional de Condiciones de Vida (ENCOVI). Guatemala.

Schifini, JP. (2005). Análisis Institucional del Sector de los Servicios de Agua Potable y del Saneamiento en Guatemala. Ciudad de Guatemala: UE-PNUD.

Solanes, M. y Jouravlev, A. (2006). Water Governance for development and sustainability. CEPAL, Serie: Recursos naturales e infraestructura. Santiago de Chile.

Tavico, V. (2010). Principales reformas 
al Código Municipal 2010. Guatemala: Promudel.

Valenzuela, E. (2007). Cien consejos para líderes del poder local apropiado. Santiago de Chile: ICHEM de la Universidad Autónoma.

(2010). Guía de Políticas Públicas Municipales. Guatemala: Promudel.

(2011). Libro Municipal del Agua. Guatemala: Promudel.

(2012). Administración Municipal Moderna en Guatemala (e). Guatemala: Promudel - Universidad Mariano Gálvez. 